\title{
Relationship between the outcome of low-dose egg oral immunotherapy and the fold-difference levels of allergen-specific IgE and IgG4 in serum
}

\author{
Akihiro Maeta, ${ }^{1}$ Yuri Takaoka, ${ }^{2}$ Makoto Kameda, ${ }^{2}$ Kyoko Takahashi ${ }^{1}$
}

\begin{abstract}
Background: There are no indices to monitor desensitization by low-dose egg oral immunotherapy (eOIT).

Objective: We aimed to examine the relationship between desensitization by low-dose eOIT and the changes in allergen-specific immunoglobulin E (IgE) and IgG4 levels.

Methods: We carried out low-dose eOIT in 31 patients with severe egg allergy in our previous two studies. After 4 months of treatment, the patients with no observed allergic symptoms in response to the open hard-boiled egg white challenge tests were classified as the negative group, and the remaining patients, the positive group. The fold-difference levels were calculated using $10 \log _{10}($ Titer after eOIT/Titer before eOIT).
\end{abstract}

Result: The 28 patients who completed eOIT with sufficient serum collected before and after eOIT were analyzed. The median fold-difference levels of ovomucoid-specific IgE in the negative and positive groups were 0.819 and 0.953 , respectively $(P=0.082)$. The median fold-difference levels of ovalbumin-specific IgG4 in the negative and positive groups were 2.01 and 1.29 , respectively $(P=0.057)$. In the receiver-operating characteristic curves, the area under the curves of fold-difference ovomucoid-specific IgE and ovalbumin-specific IgG4 were 0.701 and 0.719 , respectively. The challenge positive predictive values of fold-difference ovomucoid-specific IgE and ovalbumin-specific IgG4 were 83.8\% (cut-off point: 0.934 ) and $77.8 \%$ (cut-off point: 1.87 ), respectively. Moreover, the challenge positive predictive value in patients with both $0.934<$ ovomucoid-specific IgE and ovalbumin-specific IgG4 < 1.87 was $100 \%$.

Conclusion: The fold-difference levels of allergen-specific IgE and IgG4 in serum are considered useful for monitoring desensitization by low-dose OIT.

Key words: Hen's egg allergy, Egg oral immunotherapy (eOIT), IgE, IgG4, Receiver-operating characteristic curve, Cross tabulation

\section{From:}

${ }^{1}$ Department of Food Science and Nutrition, School of Food Science and Nutrition, Mukogawa Women's University, Hyogo, Japan

Department of Pediatrics, Osaka Prefectural Hospital Organization Osaka Habikino Medical Center, Osaka, Japan

\section{Introduction}

Oral immunotherapy (OIT) has been studied as a new treatment for food allergies. ${ }^{1}$ However, there is a risk of severe symptoms with OIT, ${ }^{2}$ and safety protocols are required for severe egg-allergic patients who cannot ingest as little as $0.5 \mathrm{~g}$ of boiled egg white (EW) without experiencing allergic symptoms. In a previous study, we developed a low-egg allergen cookie (LAC) recipe using heated EW meringue. ${ }^{3}$

\author{
Corresponding author: \\ Kyoko Takahashi \\ Department of Food Science and Nutrition, School of Food Science \\ and Nutrition, Mukogawa Women's University, \\ 6-46, Ikebiraki-cho, Nishinomiya, Hyogo, 663-8558, Japan \\ E-mail: taka@mukogawa-u.ac.jp
}

Prior to starting low-dose egg oral immunotherapy (eOIT) with hard-boiled EW in patients with severe egg allergy, we carried out low-dose eOIT using LACs. ${ }^{4,5}$ This partly increased the threshold level for hard-boiled EW. ${ }^{4,5}$ However, in eOIT using LACs, it was difficult to estimate the improvement in the egg allergy during the treatment without an oral food challenge (OFC). Therefore, we considered the need for a monitoring index of oral desensitization by low-dose OIT. 
Biomarkers related to oral desensitization by OIT are mainly the serum levels of allergen-specific immunoglobin $\mathrm{E}$ (IgE) and IgG4. IgE is the most important antibody in allergic disorders. ${ }^{1}$ IgG4 induced by allergen-specific immunotherapy can inhibit allergen-specific IgE binding to $B$ cells $^{6}$ and mast cells. ${ }^{7}$ In vitro, regulatory $\mathrm{T}$ cells abundantly produce interleukin-10, but not interleukin $-4{ }^{8}$ and interleukin-10 induced the class-switch from IgE to IgG4. ${ }^{9}$ Moreover, in a mouse model with egg allergy, eOIT induced the secretion of IL-10 and regulatory $\mathrm{T}$ cells in spleen lymphocytes. ${ }^{10}$ It is known that a decrease in allergen-specific IgE and/or an increase of allergen-specific IgG4 in the serum relates to the amelioration of food allergies using high-dose eOIT. ${ }^{11-15}$ In the study of Burk et al., ${ }^{12}$ the serum levels of egg-specific IgG4 were 4 -fold higher in the group that passed OFCs after eOIT compared with the group that did not. Moreover, Wright et al. ${ }^{13}$ and Sugimoto et al. ${ }^{14}$ reported that in patients with egg allergy, the serum levels of the allergen-specific IgG4 increased more in the group with eOIT induced desensitization than in the non-induced group. However, the cut-off values for desensitization in these studies were large, such as $5 \mathrm{~g}^{12}$ and $10 \mathrm{~g}^{13}$ of EW powder and the equivalent of one hard-boiled egg. ${ }^{14}$ Nonetheless, there are no reports on the relationship between the desensitized outcome with low-dose eOIT and the changed levels of allergen-specific IgE and IgG4 in serum. We carried out two low-dose eOIT studies ${ }^{4,5}$ and compared the changed allergen-specific IgE and IgG4 levels in serum.

\section{Methods}

This study was approved by the Ethics Committees of Osaka Habikino Medical Center (approval number: 577 and 683, UMIN number: UMIN000008012 and UMIN000014931) and Mukogawa Women's University (approval number: 11-52, 1409 , and 16-77). Written informed consent was obtained from all the patients' guardians.

\section{Clinical study protocol and participants}

The clinical study protocol and the outcome of low-dose eOIT using LACs for 4 months (UMIN000008012, UMIN00 0014931) have been described previously. ${ }^{4,5}$ The flow chart is shown in Figure 1.

Participants included children with egg allergy who, by their treating doctor's recommendation, could not receive eOIT using $0.5 \mathrm{~g}$ of hard-boiled EW (Supplementary Table 1). Before conducting eOIT, participants were screened using open hard-boiled EW challenge tests (Supplementary Table 2) and blood samples were taken (before eOIT). Symptom scores of allergic reactions were assessed according to the modified Sampson's anaphylaxis grades in the Japanese Pediatric Guideline for Food Allergy. ${ }^{16}$ Before the start of treatment, the number of pieces of LAC on the first day of eOIT was determined by the OFC with LACs (Supplementary Table 2). Low-dose eOIT was carried out for 4 months. The maximum number of LACs in a single day was ten pieces. After 4 months of treatment, open hard-boiled EW challenge tests were carried out (Supplementary Table 2) and blood samples were collected (after eOIT). The doctors identified egg-allergic patients who could ingest $3.7 \mathrm{~g}$ (final dose: $2 \mathrm{~g}$ ) of hard-boiled EW without experiencing any allergic symptoms.

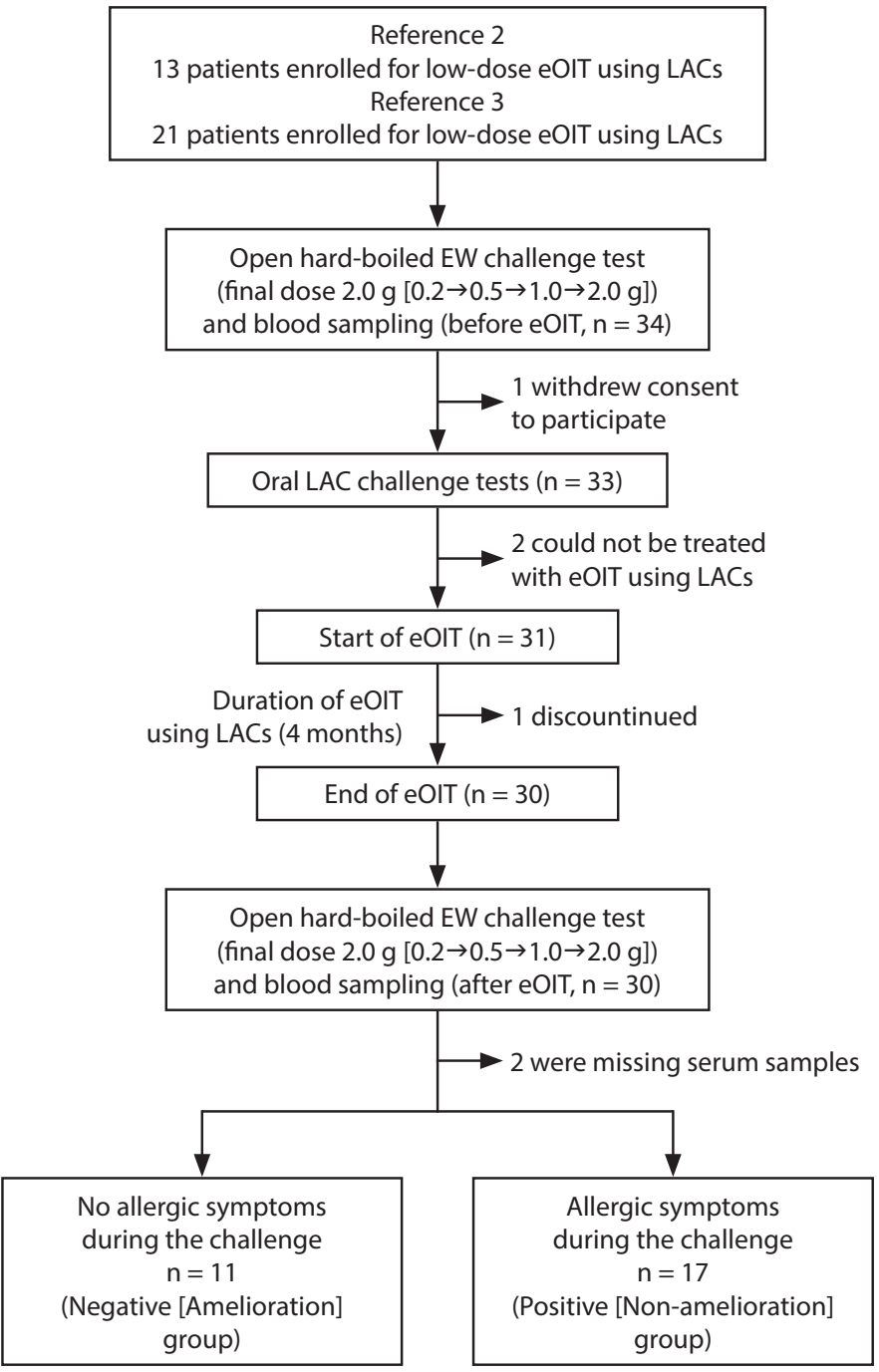

Figure 1. Flow chart of low-dose oral immunotherapy (OIT) using low-egg allergen cookies (LACs). EW, egg white.

Thus, the negative group was defined as patients who could pass the open hard-boiled EW challenge tests without experiencing any allergic symptoms after eOIT. The patients in this group showed an amelioration in the severity of their egg allergy with low-dose eOIT using LACs. The remaining patients were categorized into the positive group. Plasma samples before and after eOIT were kept frozen at $-80^{\circ} \mathrm{C}$ until analysis.

\section{Measurement of serum allergenic biomarkers}

The serum levels of EW- and ovomucoid (OM)-specific IgE were measured using the ImmunoCAP 100 instrument (Thermo Fisher Scientific, Massachusetts, USA) at the Osaka Habikino Medical Center. Serum ovalbumin (OVA)and OM-specific IgG4 levels were measured by indirect enzyme-linked immunosorbent assay at Mukogawa Women's University. ${ }^{4}$ Coating antigens $(500 \mu \mathrm{g} / \mathrm{mL})$ were used: OVA (OVA grade VI, Sigma-Aldrich Co. LLC, Missouri, USA) and $\mathrm{OM}$, which was purified from EW by the method of ethanol precipitation. ${ }^{2}$ The following secondary antibodies were used: biotin-conjugated mouse monoclonal anti-human IgG4 (Sigma-Aldrich Co., LLC, Missouri, USA) and streptavidin 
(Abcam, Cambridge, UK). We used reference human pool serum as a standard (OVA-specific IgG4; $15.3 \mu \mathrm{gA} / \mathrm{mL}$, OM-specific IgG4; $15.6 \mu \mathrm{gA} / \mathrm{mL})$. The limit of quantification (LOQ) in OVA- and OM-specific IgG4 was $0.2 \mu \mathrm{gA} / \mathrm{mL}$ and the sample determination below LOQ was $0.2 \mu \mathrm{gA} / \mathrm{mL}$.

The fold-difference levels of allergen-specific antibodies were calculated as $\log _{10}$ (Titer after eOIT) - $\log _{10}$ (Titer before eOIT $)=\log _{10}($ Titer after eOIT/Titer before eOIT $)=$ $10 \log _{10}($ Titer after eOIT/Titer before eOIT).

\section{Statistical analysis}

Data on serum levels of allergen-specific antibodies are presented as the absolute median and 25-75 percentiles. Statistical analyses were performed using the Wilcoxon signedrank test, Mann-Whitney U test, Fisher's exact test, or Chi-squared test. The familywise error rate of the t-test was corrected using the Bonferroni method $(P$-values $\times$ numbers of t-tests). $P$-values of $<0.05$ were considered to be statistically significant. The predictive accuracy of serum biomarkers related to the results of the open hard-boiled EW challenge tests after eOIT was assessed by a receiver operating characteristic (ROC) curve analysis to determine the area under the ROC curve (AUC). The cut-off values were calculated using the Youden index ${ }^{17}$ (Maxc $\{$ Sensitivity (\%) + Specificity (\%) - 100\}). All analyses were conducted using GraphPad Prism version 5.0 (La Jolla, CA, USA).

\section{Results}

Changes in the allergic biomarkers by low-dose eOIT

Analyses were performed on 28 patients who completed eOIT with sufficient serum collected before and after eOIT. The serum levels of EW- and OM-specific IgE were comparable before and after eOIT (Supplementary Table 3, $P=0.132$ and 0.164 , respectively). The serum levels of OVA- and OM-specific IgG4 significantly increased after eOIT compared to those before eOIT (Supplementary Table 3, $P=0.002$ and 0.007 , respectively). Moreover, the serum ratios of OVA-specific IgG4/EW-specific IgE and OM-specific IgG4/OM-specific IgE significantly increased after eOIT compared to those before eOIT (Supplementary Table 3, $P<0.001$ ).

\section{Changed levels of serum allergen-specific antibodies in the negative and positive groups}

From the results of the open hard-boiled EW challenge tests after eOIT, the 28 patients were divided into a negative group (amelioration, $\mathrm{n}=11$ ) and a positive group (non-amelioration, $\mathrm{n}=17$ ). Before eOIT, the serum levels of EW- and OM-specific IgE and OVA- and OM-specific IgG4 were comparable between the negative and positive groups (Table 1).

In the negative group, the serum levels of OM-specific IgE decreased (Table 1, $P=0.029$ ) and OVA-specific IgG4 increased (Table 1, $P=0.029$ ) more after eOIT than before eOIT. After eOIT, the serum levels of OVA-specific IgG4 in the negative group were significantly higher than those in the positive group (Table $1, P=0.028$ ). The serum ratios of OVA-specific IgG4/EW-specific IgE and OM-specific IgG4/ OM-specific IgE increased significantly after eOIT compared to those before eOIT (Table $1, P=0.009$ and 0.021 , respectively). In the positive group, there was no difference between the before and after eOIT serum levels of EW- and OM-specific IgE (Table 1). The serum levels of OM-specific IgG4 were higher after eOIT than before eOIT (Table 1, $P=0.040$ ). The serum ratios of OM-specific IgG4/OM-specific IgE increased significantly after eOIT compared to those before eOIT (Table $1, P=0.020)$.

Table 1. Comparisons of the serum levels of allergen-specific antibodies between before and after eOIT. ${ }^{1}$

\begin{tabular}{|c|c|c|c|c|c|c|c|c|}
\hline & \multicolumn{6}{|c|}{ Open hard-boiled EW challenge tests after eOIT } & \multirow{3}{*}{$\begin{array}{c}P \text {-value } \\
\text { Before } \\
\text { eOIT }\end{array}$} & \multirow{3}{*}{$\begin{array}{c}P \text {-value } \\
\text { After } \\
\text { eOIT }\end{array}$} \\
\hline & \multicolumn{3}{|c|}{ Negative $^{2}$ (Amelioration, $n=11$ ) } & \multicolumn{3}{|c|}{ Positive $^{2}$ (Non-amelioration, $\mathrm{n}=17$ ) } & & \\
\hline & Before eOIT & After eOIT & $p$-value ${ }^{3}$ & Before eOIT & After eOIT & $p$-value ${ }^{3}$ & & \\
\hline EW-specific IgE, $\mathrm{U}_{\mathrm{A}} / \mathrm{mL}$ & $\begin{array}{c}56.5 \\
(14.4-100)\end{array}$ & $\begin{array}{c}37.8 \\
(16.2-100)\end{array}$ & 0.234 & $\begin{array}{c}26.9 \\
(16.7-60.3)\end{array}$ & $\begin{array}{c}25.6 \\
(14.2-60.2)\end{array}$ & 1.000 & 0.967 & 1.000 \\
\hline OM-specific IgE, $\mathrm{U}_{\mathrm{A}} / \mathrm{mL}$ & $\begin{array}{c}27.7 \\
(12.1-42.2)\end{array}$ & $\begin{array}{c}16.7 \\
(11.7-39.3)\end{array}$ & 0.029 & $\begin{array}{c}12.7 \\
(7.68-47.1)\end{array}$ & $\begin{array}{c}15.2 \\
(5.39-42.0)\end{array}$ & 1.000 & 1.000 & 1.000 \\
\hline OVA-specific IgG4, $\mu \mathrm{gA} / \mathrm{mL}$ & $\begin{array}{c}1.49 \\
(0.200-3.81)\end{array}$ & $\begin{array}{c}2.68 \\
(1.25-5.89)\end{array}$ & 0.029 & $\begin{array}{c}0.230 \\
(0.200-1.78)\end{array}$ & $\begin{array}{c}0.401 \\
(0.240-1.58)\end{array}$ & 0.250 & 0.602 & 0.028 \\
\hline OM-specific IgG4, $\mu \mathrm{gA} / \mathrm{mL}$ & $\begin{array}{c}1.04 \\
(0.200-1.78)\end{array}$ & $\begin{array}{c}1.69 \\
(1.02-5.81)\end{array}$ & 0.293 & $\begin{array}{c}0.250 \\
(0.200-1.00)\end{array}$ & $\begin{array}{c}0.450 \\
(0.217-1.56)\end{array}$ & 0.040 & 0.460 & 0.100 \\
\hline OVA-sIgG4/EW-sIgE, ngA/U & $\begin{array}{c}38.3 \\
(17.6-55.1)\end{array}$ & $\begin{array}{c}58.9 \\
(16.8-158)\end{array}$ & 0.009 & $\begin{array}{c}20.2 \\
(7.75-41.9)\end{array}$ & $\begin{array}{c}25.4 \\
(9.80-77.5)\end{array}$ & 0.054 & 0.073 & 0.161 \\
\hline OM-sIgG4/OM-sIgE, ngA/ $\mathrm{U}_{\mathrm{A}}$ & $\begin{array}{c}39.8 \\
(19.2-90.0)\end{array}$ & $\begin{array}{c}103 \\
(29.9-259)\end{array}$ & 0.021 & $\begin{array}{c}32.0 \\
(10.8-55.4)\end{array}$ & $\begin{array}{c}51.5 \\
(15.1-96.5)\end{array}$ & 0.020 & 1.000 & 0.245 \\
\hline
\end{tabular}

${ }^{1}$ Data displayed median and 25-75 percentile value. $P$-values of $<0.05$ were considered statistically significant.

${ }^{2}$ The negative (amelioration) group was defined as patients who could pass the open hard-boiled EW challenge tests without allergic symptoms after eOIT. Patients in the negative group improved the severity of egg allergy by low-dose eOIT using low-egg allergen cookies (LACs). The remaining patients were categorized into the positive (non-amelioration) group.

${ }^{3}$ Statistical differences were assessed using the Wilcoxon signed-rank test (before eOIT vs. after eOIT) and Bonferroni correction $(P$-values $\times 3)$.

${ }^{4}$ Statistical differences were assessed using the Mann-Whitney $\mathrm{U}$ test (negative vs. positive) and Bonferroni correction $(P$-values $\times 3)$.

eOIT: egg oral immunotherapy; EW: egg white; OM: ovomucoid; OVA: ovalbumin 
A EW-specific IgE

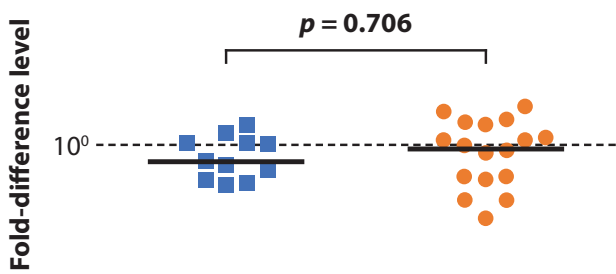

$10^{-1}$

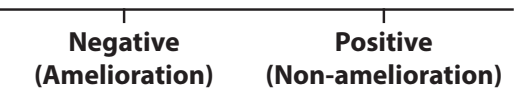

Open hard-boiled EW challenge after eOIT

C $10^{2}$

OVA-specific IgG4

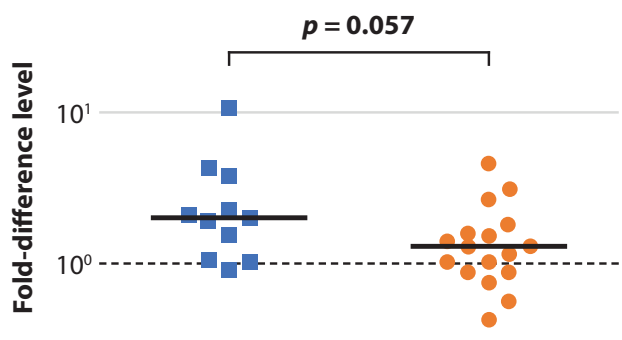

$10^{-1}$

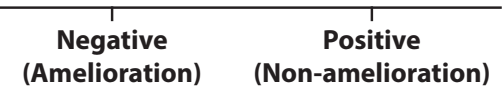

Open hard-boiled EW challenge after eOIT

E $10^{2}$ OVA-slgG4/EW-slgE

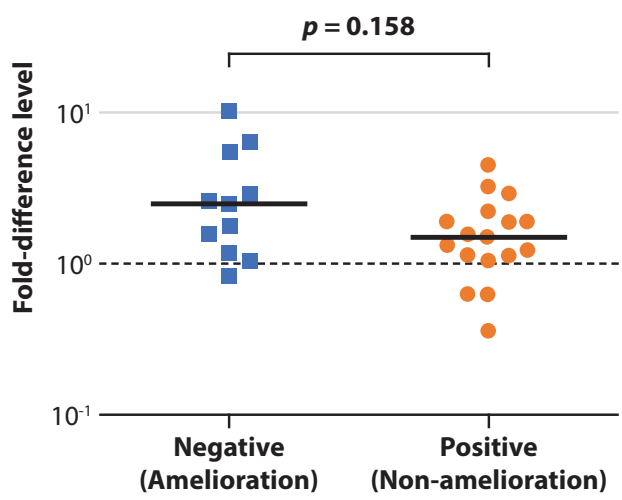

Open hard-boiled EW challenge after eOIT
B OM-specific IgE

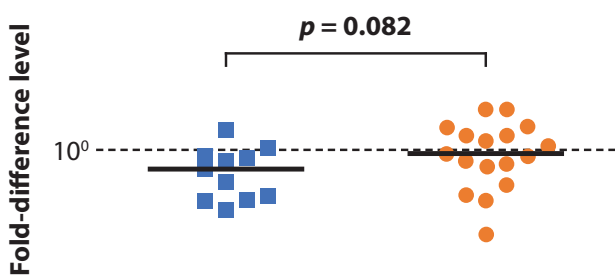

$10^{-1}$

$\begin{array}{cc}\text { Negative } & \text { Positive } \\ \text { (Amelioration) } & \text { (Non-amelioration) }\end{array}$

Open hard-boiled EW challenge after eOIT

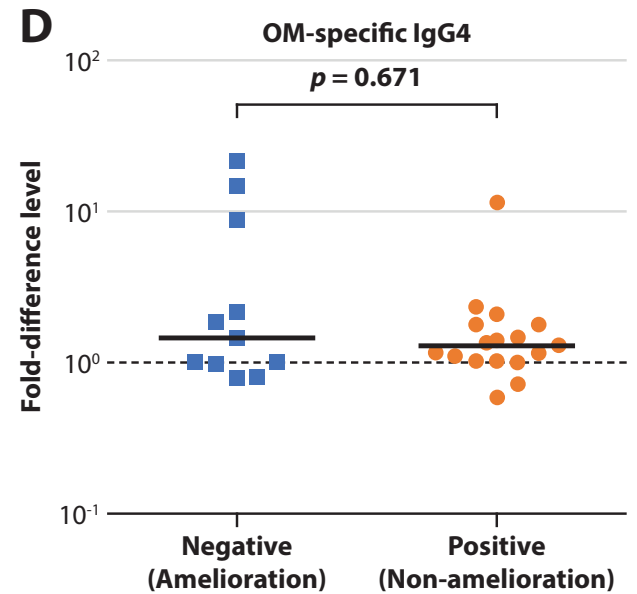

Open hard-boiled EW challenge after eOIT
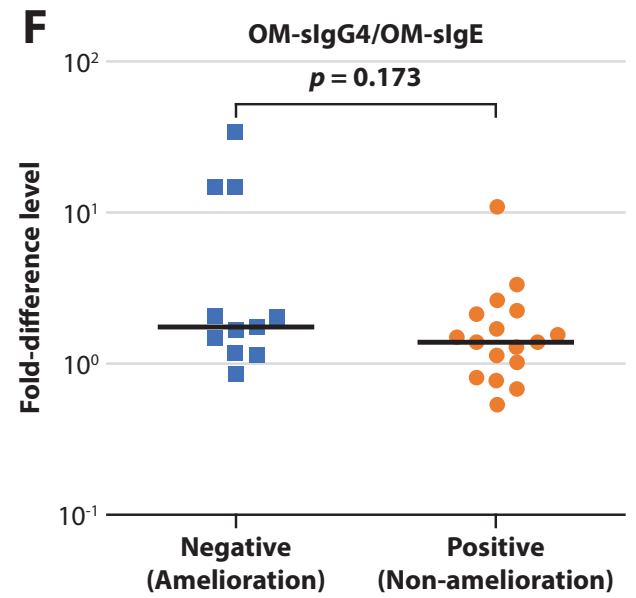

Open hard-boiled EW challenge after eOIT

Figure 2. Comparisons of the fold-difference levels of allergen-specific antibodies in serum between the negative and positive groups.

The negative (Amelioration) group was defined as patients who could pass the open hard-boiled egg white (EW) challenge tests without allergic symptoms after egg low-dose oral immunotherapy (eOIT). Patients in the negative group improved the severity of egg allergy by low-dose eOIT using low-egg allergen cookies (LAC). The other patients were categorized into the positive (non-amelioration) group. The data displayed fold-difference levels $\left(10^{\log _{10}(\text { Titer after eOIT/Titer before eOIT) }}\right)$. Statistical differences were assessed using the Mann-Whitney $U$ test. $P$-values of $<0.05$ were considered statistically significant. 

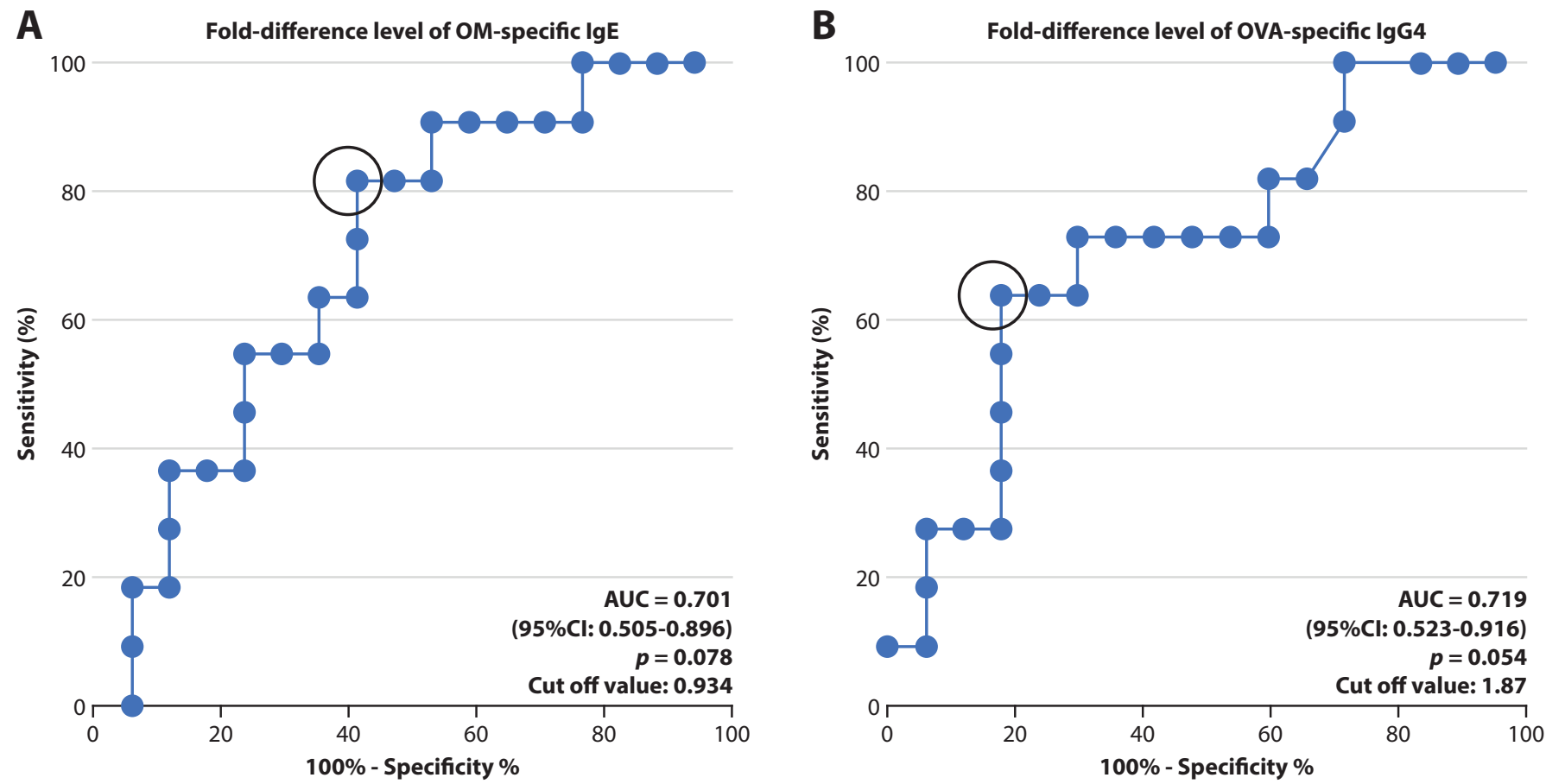

Figure 3. Receiver operating characteristic (ROC) curve of the fold-difference levels of ovomucoid (OM)-specific IgE (A) and ovalbumin (OVA)-specific IgG4 (B)

Open circles in each graph show the point of the cut-off value. The cut-off values were calculated using the Youden index ${ }^{17}$ (Maxc $\{$ Sensitivity $(\%)+$ Specificity $(\%)-100\})$.

The fold-difference levels of EW-specific IgE and OM-specific IgG4 in serum were comparable between the negative and positive groups (Figure $\mathbf{2 A}$ and $2 \mathrm{D}$ ). The fold-difference levels of the serum ratios of OVA-specific IgG4/EW-specific IgE and OM-specific IgG4/OM-specific IgE were not significantly different between the negative and positive groups (Figure 2E and 2F). The median fold-difference serum levels of OM-specific IgE in the negative and positive groups were 0.819 and 0.953 , respectively (Figure $2 \mathrm{~B}, P=0.082$ ). Moreover, the median fold-difference serum levels of OVA-specific IgG4 in the negative and positive groups were 2.01 and 1.29, respectively (Figure 2C, $P=0.057$ ).

Predictive accuracy of fold-difference OM-specific IgE and OVA-specific IgG4 for the results of the open hard-boiled EW challenge tests after eOIT

The fold-difference levels of OM-specific IgE indicated moderate accuracy for the relationship with the result of open hard-boiled EW challenge tests after eOIT (Figure 3A, AUC $=0.703$ (95\% confidence interval: 0.505-0.896), $P=0.078$ ). Moreover, the fold-difference levels of OVA-specific IgG4 indicated moderate accuracy for the relationship with the result of open hard-boiled EW challenge tests after eOIT (Figure 3B, AUC $=0.719$ (95\% confidence interval: 0.523-0.916), $P=0.054)$. The cut-off values of the fold-difference levels of OM-specific IgE and OVA-specific IgG4 were 0.934 and 1.87, respectively (Figure $\mathbf{3 A}$ and $\mathbf{3 B}$ ).$$
15
$$

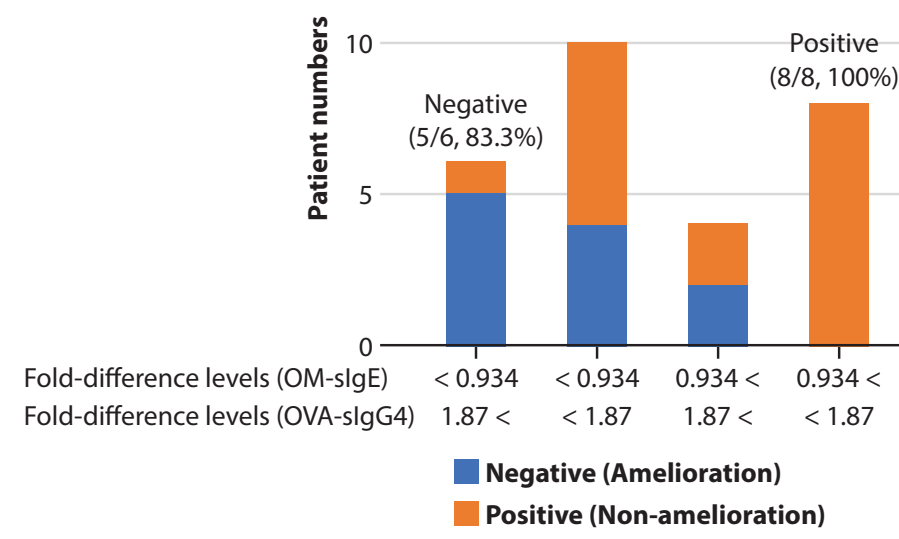

Figure 4. Relationship of the result of open hard-boiled egg white (EW) challenge tests after low dose egg oral immunotherapy (eOIT) and the fold-difference levels of ovomucoid-specific IgE (OM-sIgE) and ovalbumin-specific IgG4 (OVA-sIgE).

Date displayed patient numbers. The negative (Amelioration) group was defined as patients who could pass the open hardboiled egg white (EW) challenge tests without allergic symptoms after eOIT. Patients in the negative group improved the severity of egg allergy by low-dose eOIT using low-egg allergen cookies (LAC). The remaining patients were categorized into the positive (non-amelioration) group. The cut-off values were calculated using the Youden index ${ }^{17}$ (Maxc \{Sensitivity $(\%)+$ Specificity $(\%)-100\})$. 
For the fold-difference levels of OM-specific $\operatorname{IgE}$ and OVA-specific IgG4, we carried out a $2 \times 2$ cross tabulation using the cut-off values calculated from the ROC curve (Supplementary Table 4). In the cut-off value of the fold-difference levels of OM-specific IgE, the EW challenge negative predictive value and EW challenge positive predictive value were $56.3 \%$ and $83.0 \%$, respectively (Supplementary Table 4 ). In the cut-off value of the fold-difference levels of OVA-specific IgG4, the EW challenge negative predictive value and EW challenge positive predictive value were $70.0 \%$ and $77.8 \%$, respectively (Supplementary Table 4 ). In the $2 \times 4$ cross tabulation (Supplementary Table 5), the EW challenge negative predictive value in the patients with both OM-specific IgE < 0.934 and 1.87 < OVA-specific IgG4 was $83.3 \%$ (5/6) (Figure 4). The EW challenge positive predictive value in patients with both $0.934<$ OM-specific IgE and OVA-specific IgG4 < 1.87 was $100 \%(8 / 8)$ (Figure 4). Moreover, OM-specific IgE $<0.934$ and/or $1.87<$ OVA-specific IgG4 had a specificity of 94\% (16/17) and $0.934<$ OM-specific IgE and/or OVA-specific IgG4 $<1.87$ had a sensitivity of $100 \%(11 / 11)$ (Supplementary Table 5).

\section{Discussion}

In the egg-allergic patients treated with low-dose eOIT, the serum levels of allergen-specific IgE decreased and allergen-specific IgG4 increased. ${ }^{18}$ Moreover, the changed levels of these antibodies were larger in the patients that had an improvement in the severity of their food allergy. ${ }^{13,14}$ We observed similar changes in this current study. Therefore, we considered that the low-dose eOIT using LACs for patients with severe egg allergy-induced immunological responses similar to those in previous studies.

Previous studies ${ }^{12-14,18}$ suggested that the serum egg allergen-specific IgG4 levels following eOIT increase immediately after the start of the treatment and then plateau during the treatment. In contrast, the decrease in egg allergen-specific IgE levels in serum following eOIT is slower than the increase in egg allergen-specific IgG4 levels..$^{12-14,18}$ Moreover, Wright et al. ${ }^{13}$ suggested that OM is more susceptible to OIT than OVA. In this study, the fold-difference levels of OM-specific IgE in serum decreased more in the negative group than in the positive group. Furthermore, the serum levels of OVA-specific IgG4 increased more in the negative group than in the positive group. From the analysis of $2 \times 2$ cross tabulation, both the sensitivity in the fold-difference levels of OM-specific $\operatorname{IgE}(81.8 \%)$ and the specificity in the fold-difference levels of OVA-specific IgG4 were high (82.4\%). We considered that the changes in egg allergen-specific antibodies by eOIT were not uniform.

In this study, the EW challenge-negative predictive values in the fold-difference levels of OM-specific IgE and OVA-specific IgG4 were not high (56.3\% and $70.0 \%$, respectively). However, in the $2 \times 4$ cross tabulation, the EW challenge-negative predictive value in patients with both OM-specific IgE $<$ 0.934 and 1.87 < OVA-specific IgG4 was $83.3 \%(5 / 6)$. Therefore, we showed that if both the decrease in the OM-specific IgE and the increase in the OVA-specific IgG4 were large, the chances of improvement of egg allergy by low-dose eOIT are high. In contrast, the EW-challenge-positive predictive values in the fold-difference levels of OM-specific IgE and OVA-specific IgG4 were high $(83.0 \%$ and $77.8 \%$, respectively). Moreover, the EW-challenge-positive predictive value in patients with both $0.934<$ OM-specific IgE and OVA-specific IgG4 $<1.87$ was $100 \%(8 / 8)$. Thus, we indicated that if both the decrease in the OM-specific IgE and the increase in the OVA-specific IgG4 are small, the chances of improvement in egg allergy using low-dose eOIT are low. We speculated that if the immuno-stimulation due to the intake of allergen was weak, the treatment effects of OIT would be small, and thus, the fold-difference levels of allergen-specific antibodies in serum could be a good biomarker to estimate allergen stimulation by OIT. Therefore, we considered that the treatment outcome of low-dose eOIT could be attributed to the fold-difference levels of some allergen-specific antibodies.

Our study has several limitations. The number of patients in the negative and positive groups was not equal, and the total number of patients was small. The treatment period in this experiment was shorter than that in previous OIT studies. ${ }^{12-15,17}$ Because the amount of serum collected was small, we could not comprehensively measure the serum levels of antigen-specific antibodies. There was no index that was more than 0.9 in the AUC, and the biomarkers in this experiment cannot be used to determine low-dose eOIT outcomes instead of OFC.

In conclusion, the serum levels of OM-specific IgE decreased and OVA-specific IgG4 increased in the improved egg-allergic patients by low-dose eOIT using LACs. The EW challenge-negative predictive value and EW challenge positive predictive value combining these fold-difference levels were high $(83.3 \%$ and $100 \%$, respectively [Supplementary Table 6]). Therefore, we consider that the fold-difference level of OM-specific IgE and OVA-specific IgG4 in serum is useful for monitoring oral desensitization induced by low-dose eOIT in patients with severe egg allergy.

\section{Conflicts of interest}

The authors have no conflicts of interest to declare.

\section{Funding}

This research was supported (exploration of serum index to predict treatment outcome in oral immunotherapy) by a Grant for Young Scientists of the Japan Society of Nutrition and Food Science in 2019.

\section{Author contributions}

- AM, YT, MK, and KT designed the study.

- YT and MK mainly diagnosed children with allergies and recruited the study participants.

- AM and KT analyzed serum samples at Mukogawa Women's University.

- AM and YT drafted the manuscript.

- All authors have read and approved the final version of the manuscript. 


\section{Acknowledgments}

We extend our sincerest gratitude to the children who participated in the study and their guardians. Additionally, we are grateful to the students of Mukogawa Women's University (namely, Yuka Yokoyama, Mako Inoue, Hiroka Tsutsui, Ayano Imao, Megumi Sumie, and Marin Matsushima) for their contribution to this work as well as the members (Shinichi Takahashi, Takahiro Muroya, Amane Shigekawa, Yuki Tsurinaga, Norihito Iba, Yukinori Yoshida, and Satoru Doi) of the division of pediatrics at the Osaka Habikino Medical Center. Yoichi M. Ito provided the allocation software and calculated the sample size. Moreover, we would like to thank Editage for the English language editing.

\section{References}

1. Ebisawa M, Ito K, Fujisawa T. Food Allergy Committee, Japanese Society of Pediatric Allergy and Clinical Immunology. Japanese pediatric guideline for food allergy 2016. Tokyo: Kyowa Kikaku Ltd; 2016.

2. Nurmatov U, Dhami S, Arasi S, Pajno GB, Fernandez-Rivas M, Muraro A, et al. Allergen immunotherapy for IgE-mediated food allergy: a systematic review and meta-analysis. Allergy. 2017;72:1133-47.

3. Takahashi K, inventor; Mukogawa Gakuin., assignee. The method for producing a hypoallergenic egg white and manufacturing low egg allergenic food. Japan published unexamined patent JP2007259805. 2007 Oct 11.

4. Maeta A, Matsushima M, Muraki N, Asano M, Takaoka Y, Kameda M, et al. Low-dose oral immunotherapy using low-egg-allergen cookies for severe egg-allergic children reduces allergy severity and affects allergen-specific antibodies in serum. Int Arch Allergy Immunol. 2018;175:70-6.

5. Takaoka Y, Maeta A, Takahashi K, M Ito Y, Takahashi S, Muroya T, et al. Effectiveness and safety of double-blind, placebo-controlled, low-dose oral immunotherapy with low allergen egg-containing cookies for severe hen's egg allergy: a single-center analysis. Int Arch Allergy Immunol. 2019;180:244-9.

6. Nouri-Aria KT, Wachholz PA, Francis JN, Jacobson MR, Walker SM, Wilcock LK, et al. Grass pollen immunotherapy induces mucosal and peripheral IL-10 responses and blocking IgG activity. J Immunol. 2004; 172:3252-9.

7. Santos AF, James LK, Bahnson HT, Shamji MH, Couto-Francisco NC, Islam S, et al. IgG4 inhibits peanut-induced basophil and mast cell activation in peanut-tolerant children sensitized to peanut major allergens. J Allergy Clin Immunol. 2015;135:1249-56.

8. Groux H, O'Garra A, Bigler M, Rouleau M, Antonenko S, de Vries JE, Roncarolo MG. A CD4+ T-cell subset inhibits antigen-specific T-cell responses and prevents colitis. Nature. 1997;389:737-42.

9. Jeannin P, Lecoanet S, Delneste Y, Gauchat JF, Bonnefoy JY. IgE versus IgG4 production can be differentially regulated by IL-10. J Immunol. 1998;160:3555-61.

10. Maeta A, Matsushima M, Katahira R, Sakamoto N, Takahashi K. Diets supplemented with $1 \%$ egg white induce oral desensitization and immune tolerance in an egg white-specific allergic mouse model. Int Arch Allergy Immunol. 2018;176:205-14.

11. Caminiti L, Pajno GB, Crisafulli G, Chiera F, Collura M, Panasci G, et al. Oral immunotherapy for egg allergy: a double-blind placebo-controlled study, with post desensitization follow-up. J Allergy Clin Immunol Pract. 2015;3:532-9.
12. Burks AW, Jones SM, Wood RA, Fleischer DM, Sicherer SH, Lindblad RW, et al. Oral immunotherapy for treatment of egg allergy in children. N Engl J Med. 2012;367233-43.

13. Wright BL, Kulis M, Orgel KA, Burks AW, Dawson P, Henning AK, et al. Component-resolved analysis of IgA, IgE, and IgG4 during egg OIT identifies markers associated with sustained unresponsiveness. Allergy. 2016;71:1552-60.

14. Sugimoto M, Kamemura N, Nagao M, Irahara M, Kagami S, Fujisawa T, Kido H. Differential response in allergen-specific IgE, IgGs, and IgA levels for predicting outcome of oral immunotherapy. Pediatr Allergy Immunol. 2016;27:276-82.

15. Andorf S, Bunning B, Tupa D, Cao S, Long AJ, Borre MP, et al. Trends in egg specific immunoglobulin levels during natural tolerance and oral immunotherapy. Allergy. 2020;75:1454-6.

16. Urisu A, Kondo N. Japanese Society of Pediatric Allergy and Clinical Immunology (ed). Japanese Pediatric Guideline for Food Allergy 2012. Tokyo: Kyowa Kikaku Ltd; 2012.

17. Youden, WJ. Index for rating diagnostic tests. Cancer. 1950;3:32-5.

18. Yanagida N, Sato S, Asaumi T, Nagakura K, Ogura K, Ebisawa M. Safety and efficacy of low-dose oral immunotherapy for hen's egg allergy in children. Int Arch Allergy Immunol. 2016;171:265-8.

\section{Supplementary Table 1. Demographic data before eOIT ${ }^{1}$}

\begin{tabular}{lc|}
\multicolumn{1}{c}{ Items } & eOIT $(\mathbf{n}=\mathbf{2 8})$ \\
\hline Age & 6 \\
Sex (Male:Female) & $22: 6$ \\
\hline Food allergies other than for egg & \\
\hline Milk & 11 \\
Wheat & 1 \\
Peanuts & 4 \\
\hline Others & 7 \\
\hline
\end{tabular}

Number of food allergies

1 (only egg) 11

2

11

more 3

Other allergic disorders

Atopic dermatitis 20

Allergic rhinitis

Allergic conjunctivitis

Bronchial asthma

12

${ }^{1}$ Data of age showed median, and of the other items displayed numbers. eOIT: egg oral immunotherapy 
Supplementary Table 2. Results of open hard-boiled egg white test before and after eOIT

\begin{tabular}{|c|c|c|c|c|c|c|c|c|c|c|c|c|c|c|}
\hline \multirow{3}{*}{$\begin{array}{c}\text { Patient } \\
\text { ID }\end{array}$} & \multicolumn{6}{|c|}{ Before eOIT } & \multirow{3}{*}{$\begin{array}{c}\text { Numbers of } \\
\text { pieces of LAC at } \\
\text { the first day of } \\
\text { eOIT }^{3}\end{array}$} & \multicolumn{6}{|c|}{ After eOIT } & \multirow{3}{*}{ Reference } \\
\hline & \multirow{2}{*}{$\begin{array}{c}\text { Final } \\
\text { Dose }(\mathbf{g})^{1}\end{array}$} & \multicolumn{5}{|c|}{ Symptom scores $^{2}$} & & \multirow{2}{*}{$\begin{array}{c}\text { Final } \\
\text { Dose }(g)^{1}\end{array}$} & \multicolumn{5}{|c|}{ Symptom scores $^{2}$} & \\
\hline & & $\mathbf{S}$ & G & $\mathbf{R}$ & C & $\mathbf{N}$ & & & $\mathbf{S}$ & G & $\mathbf{R}$ & C & $\mathbf{N}$ & \\
\hline eO-1 & 1.0 & 0 & 2 & 0 & 0 & 0 & 2 & 2.0 & 0 & 0 & 0 & 0 & 0 & 2 \\
\hline $\mathrm{eO}-2$ & 1.0 & 0 & 2 & 0 & 0 & 0 & 10 & 2.0 & 0 & 2 & 0 & 0 & 0 & 2 \\
\hline $\mathrm{eO}-3$ & 0.2 & 0 & 2 & 0 & 0 & 0 & 1 & 2.0 & 0 & 0 & 0 & 0 & 0 & 2 \\
\hline $\mathrm{eO}-4$ & 0.2 & 0 & 0 & 2 & 0 & 0 & 1 & 2.0 & 0 & 0 & 0 & 0 & 0 & 2 \\
\hline $\mathrm{eO}-5$ & 0.5 & 0 & 3 & 0 & 0 & 0 & 2 & 2.0 & 0 & 0 & 0 & 0 & 0 & 2 \\
\hline $\mathrm{eO}-6$ & 1.0 & 0 & 2 & 0 & 0 & 2 & 10 & 2.0 & 0 & 0 & 0 & 0 & 0 & 2 \\
\hline $\mathrm{eO}-7$ & 1.0 & 0 & 2 & 0 & 0 & 0 & 10 & 2.0 & 0 & 2 & 0 & 0 & 0 & 2 \\
\hline $\mathrm{eO}-8$ & 1.0 & 1 & 2 & 2 & 0 & 0 & 1 & 2.0 & 0 & 2 & 2 & 0 & 0 & 2 \\
\hline eO-9 & 0.1 & 0 & 2 & 0 & 0 & 0 & 1 & 1.0 & 0 & 2 & 4 & 3 & 0 & 2 \\
\hline eO-10 & 2.0 & 2 & 3 & 0 & 0 & 0 & 1 & 2.0 & 2 & 2 & 3 & 0 & 0 & 2 \\
\hline eO-11 & 1.0 & 2 & 0 & 0 & 0 & 0 & 1 & 1.0 & 1 & 2 & 2 & 0 & 0 & 2 \\
\hline eO-12 & 0.5 & 0 & 2 & 0 & 0 & 0 & 10 & 1.0 & 0 & 2 & 0 & 0 & 0 & 3 \\
\hline eO-13 & 0.5 & 0 & 2 & 0 & 0 & 0 & 10 & 1.0 & 0 & 3 & 0 & 0 & 2 & 3 \\
\hline eO-14 & 0.5 & 0 & 2 & 0 & 0 & 0 & 10 & 1.0 & 0 & 2 & 0 & 0 & 0 & 3 \\
\hline eO-15 & 1.0 & 0 & 2 & 0 & 0 & 0 & 10 & 2.0 & 0 & 0 & 0 & 0 & 0 & 3 \\
\hline eO-16 & 2.0 & 0 & 0 & 3 & 0 & 0 & 10 & 2.0 & 0 & 0 & 0 & 0 & 0 & 3 \\
\hline eO-17 & 0.5 & 0 & 3 & 0 & 3 & 0 & 2 & 0.2 & 0 & 2 & 0 & 0 & 0 & 3 \\
\hline eO-18 & 1.0 & 0 & 2 & 0 & 0 & 0 & 2 & 0.5 & 0 & 2 & 0 & 0 & 0 & 3 \\
\hline eO-19 & 1.0 & 2 & 0 & 4 & 3 & 0 & 10 & 0.2 & 0 & 0 & 3 & 0 & 0 & 3 \\
\hline eO-20 & 2.0 & 2 & 0 & 0 & 3 & 0 & 10 & 2.0 & 0 & 0 & 0 & 0 & 0 & 3 \\
\hline eO-21 & 1.0 & 0 & 2 & 0 & 0 & 0 & 4 & 1.0 & 0 & 2 & 2 & 0 & 0 & 3 \\
\hline eO-22 & 1.0 & 2 & 0 & 0 & 3 & 0 & 5 & 0.5 & 1 & 1 & 0 & 0 & 0 & 3 \\
\hline eO-23 & 1.0 & 2 & 0 & 2 & 0 & 0 & 10 & 1.0 & 1 & 2 & 2 & 3 & 0 & 3 \\
\hline eO-24 & 0.5 & 0 & 1 & 0 & 0 & 0 & 2 & 2.0 & 0 & 0 & 0 & 0 & 0 & 3 \\
\hline eO-25 & 1.0 & 0 & 2 & 0 & 0 & 0 & 10 & 2.0 & 0 & 0 & 0 & 0 & 0 & 3 \\
\hline eO-26 & 0.1 & 0 & 2 & 0 & 0 & 0 & 5 & 1.0 & 0 & 2 & 0 & 0 & 0 & 3 \\
\hline eO-27 & 1.0 & 2 & 0 & 2 & 0 & 0 & 10 & 2.0 & 2 & 0 & 0 & 0 & 0 & 3 \\
\hline eO-28 & 0.5 & 0 & 3 & 0 & 0 & 0 & 10 & 2.0 & 0 & 0 & 0 & 0 & 0 & 3 \\
\hline
\end{tabular}

${ }^{1}$ Patients were administered $0.2 \mathrm{~g}, 0.5 \mathrm{~g}, 1.0 \mathrm{~g}$, and $2.0 \mathrm{~g}$ of hard-boiled EW at 20 -min intervals, the results of which were determined by the treating doctor.

${ }^{2}$ Symptom scores indicated the regions of reaction (S: skin symptoms; G: gastrointestinal tract symptoms; R: respiratory tract symptoms; C: cardiovascular symptoms; $\mathrm{N}$ : neurological symptoms) and severity grades (1-5) according to the modified Sampson's anaphylaxis grades in the Japanese Pediatric Guideline for Food Allergy $2012 .{ }^{16}$ The results of the challenge were determined by the treating doctor.

${ }^{3}$ Participants were administered $1,2,4$, and 10 pieces at LAC at 20-min intervals, the results of which were determined by the treating doctor. eOIT: egg oral immunotherapy 
Supplementary Table 3. Comparison of the serum levels of allergen-specific antibodies before and after eOIT $^{1}$

\begin{tabular}{lccc} 
& Before eOIT & After eOIT & p-value $^{2}$ \\
\hline Serum antibodies & & & \\
\hline EW-specific IgE, $\mathrm{U}_{\mathrm{A}} / \mathrm{mL}$ & $28.5(15.9-72.8)$ & $28.7(16.7-75.3)$ & 0.132 \\
\hline OM-specific IgE, $\mathrm{U}_{\mathrm{A}} / \mathrm{mL}$ & $19.6(0.98-45.2)$ & $16.0(8.44-38.9)$ & 0.164 \\
OVA-specific IgG4, $\mathrm{ggA} / \mathrm{mL}$ & $0.399(0.200-2.17)$ & $1.02(0.334-2.85)$ & 0.002 \\
OM-specific IgG4, $\mu \mathrm{gA} / \mathrm{mL}$ & $0.442(0.200-1.63)$ & $0.834(0.255-2.53)$ & 0.007 \\
OVA-sIgG4/EW-sIgE, ngA/ $\mathrm{U}_{\mathrm{A}}$ & $28.8(9.02-51.9)$ & $30.2(13.3-106)$ & $<0.001$ \\
OM-sIgG4/OM-sIgE, ngA/ $\mathrm{U}_{\mathrm{A}}$ & $34.6(17.5-65.7)$ & $55.3(23.3-134)$ & $<0.001$ \\
\hline
\end{tabular}

${ }^{1}$ Data displayed median and 25-75 percentile value $(n=28)$.

${ }^{2}$ Statistical differences in the challenge test and serum levels were assessed using the Wilcoxon signed rank test. $P$-values of $<0.05$ were considered statistically significant.

eOIT: egg oral immunotherapy; EW: egg white; OM: ovomucoid; OVA: ovalbumin

Supplementary Table 4 . A $2 \times 2$ cross tabulation of the fold-difference levels of OM-specific IgE and OVA-specific IgG4 in serum $^{1}$

\begin{tabular}{|c|c|c|c|c|c|c|c|}
\hline & \multicolumn{3}{|c|}{ Open hard-boiled EW challenge tests after eOIT } & \multirow[b]{2}{*}{$\begin{array}{c}\text { Sensitivity } \\
(95 \% \mathrm{CI})\end{array}$} & \multirow[b]{2}{*}{$\begin{array}{c}\text { Specificity } \\
(95 \% \mathrm{CI})\end{array}$} & \multirow[b]{2}{*}{$\begin{array}{c}\text { EW challenge } \\
\text { negative predictive } \\
\text { value }(95 \% \mathrm{CI})\end{array}$} & \multirow[b]{2}{*}{$\begin{array}{l}\text { EW challenge } \\
\text { positive predictive } \\
\text { value }(95 \% \mathrm{CI})\end{array}$} \\
\hline & $\begin{array}{c}\text { Negative }^{2} \\
(\text { Amelioration) } \\
(\mathbf{n}=\mathbf{1 1})\end{array}$ & $\begin{array}{c}\text { Positive }^{2} \\
\text { (Non-amelioration) } \\
(\mathbf{n}=17)\end{array}$ & $p$-value ${ }^{3}$ & & & & \\
\hline \multicolumn{8}{|c|}{ OM-specific IgE (cut-off value: 0.934 ) } \\
\hline$<0.934$ & 9 & 7 & \multirow{2}{*}{0.054} & \multirow{2}{*}{$\begin{array}{l}81.8 \%, 9 / 11 \\
(48.2-97.7)\end{array}$} & \multirow{2}{*}{$\begin{array}{c}58.8 \%, 10 / 17 \\
(32.9-81.6)\end{array}$} & \multirow{2}{*}{$\begin{array}{l}56.3 \%, 9 / 16 \\
(29.9-80.3)\end{array}$} & \multirow{2}{*}{$\begin{array}{c}83.0 \%, 10 / 12 \\
(52.0-97.9)\end{array}$} \\
\hline $0.934<$ & 2 & 10 & & & & & \\
\hline \multicolumn{8}{|c|}{ OVA-specific IgG4 (cut-off value: 1.87) } \\
\hline $1.87<$ & 7 & 3 & \multirow{2}{*}{0.020} & \multirow{2}{*}{$\begin{array}{l}63.6 \%, 7 / 11 \\
(30.8-89.1)\end{array}$} & \multirow{2}{*}{$\begin{array}{c}82.4 \%, 14 / 17 \\
(56.6-96.2)\end{array}$} & \multirow{2}{*}{$\begin{array}{l}70.0 \%, 7 / 10 \\
(34.8-93.3)\end{array}$} & \multirow{2}{*}{$\begin{array}{c}77.8 \%, 14 / 18 \\
(52.4-93.6)\end{array}$} \\
\hline$<1.87$ & 4 & 14 & & & & & \\
\hline
\end{tabular}

${ }^{1}$ The cut-off values of the fold-difference levels ( $\left.10^{\text {Log }_{10}(\text { Titer after eorr/Titer before eorT) }}\right)$ of OM-specific IgE and OVA-specific IgG4 were calculated from receiver operating characteristic (ROC) curves using the Youden index ${ }^{17}$ (Figure 3A and 3B, respectively).

${ }^{2}$ The negative group (amelioration) was defined as patients who could pass the open hard-boiled EW challenge tests without allergic symptoms after eOIT. Patients in the negative group improved the severity of egg allergy by low-dose eOIT using LACs. The remaining patients were categorized into the positive (non-amelioration) group.

${ }^{3}$ Statistical differences were assessed using Fisher's exact test. $P$-values of $<0.05$ were considered statistically significant.

CI: confidence interval; eOIT: egg oral immunotherapy; EW: egg white; OM: ovomucoid; OVA: ovalbumin

Supplementary Table 5. A $2 \times 4$ cross tabulation of the fold-difference levels of OM-specific IgE and OVA-specific IgG4 in serum $^{1}$

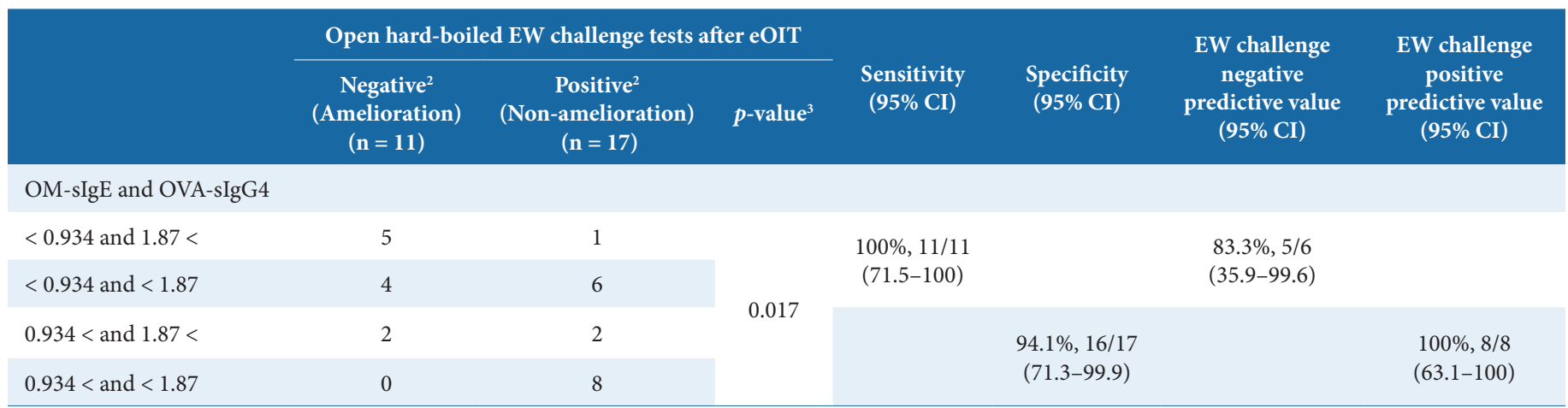

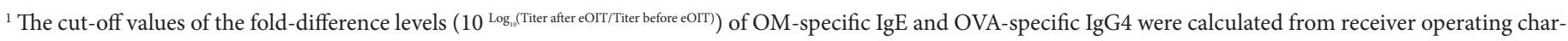
acteristic (ROC) curves using the Youden index ${ }^{17}$ (Figure 3A and 3B, respectively).

${ }^{2}$ The negative group (amelioration) was defined as patients who could pass the open hard-boiled EW challenge tests without allergic symptoms after eOIT. Patients in the negative group improved the severity of egg allergy by low-dose eOIT using LACs. The remaining patients were categorized into the positive (non-amelioration) group.

${ }^{3}$ Statistical differences were assessed using the chi-square $\left(\chi^{2}\right)$ test. $P$-values of $<0.05$ were considered statistically significant.

CI: confidence interval; eOIT: egg oral immunotherapy; EW: egg white; OM: ovomucoid; OVA: ovalbumin 